\title{
WHAT IF CRONBACH WAS RIGHT?
}

Caroline A. Mathieu, Université Laval, Québec, Canada

Cynthia Mathieu, Université du Québec à Trois-Rivières, Québec, Canada

$\underline{\text { dx.doi.org/10.18374/EJM-13-1.8 }}$

\begin{abstract}
In quantitative research, an important principle is data objectivity, which allows the generalization of the thesis. Cronbach thinks that the empirical generalization of social sciences stems from the failure of the research strategy (Cronbach, 1982). This research will verify if abstraction of the contexts should be made or not during the generalization of the results. A comparison between the results of a questionnaire managed with resident doctors at the University of Sherbrooke in 2007 and the results of the same questionnaire with 605 resident doctors of Quebec in 2011 will be carried out. In this research, three contexts were evaluated (time, place of practice and type of practice) and only the place of practice context affected the generalization of the results between the 2007 and 2011 data.
\end{abstract}

Keywords: Statistics, Context in research, Generalization, Medical residentsUniformity in the physical apparatus of life wouldbe no engraves matter, goal uniformity in mattersof thought and opinion is much more dangerous(Russell, 2004). 